Content list available at: https:/openbiomedicalengineeringjournal.com
Biomedical
lournal

RESEARCH ARTICLE

\title{
Reliability and Validity of the Feedback Sensor for Activating the Transversus Abdominis Muscle
}

Duangruedee Dissanguan ${ }^{1}$, Patraporn Sitilertpisan ${ }^{1, *}$, Suchart Kiatwattanacharoen ${ }^{2}$, Leonard H. Joseph ${ }^{3}$, Pinyo Puangmali ${ }^{4}$ and Aatit Paungmali, ${ }^{1, *}$

'Department of Physical Therapy, Faculty of Associated Medical Sciences, Chiang Mai University, Chiang Mai, Thailand

${ }^{2}$ Department of Radiologic Technology, Faculty of Associated Medical Sciences, Chiang Mai University, Chiang Mai, Thailand

${ }^{3}$ School of Health Science, University of Brighton, East, Sussex, United Kingdom

${ }^{4}$ Department of Mechanical Engineering, Faculty of Engineering, Chiang Mai University, Chiang Mai, Thailand

\section{Abstract: \\ Background:}

Core muscle activation is an effective intervention for the management of Low Back Pain (LBP). This study developed new feedback for detecting activation of the transversus abdominis muscle in the lumbar spine. The purpose of this study was to examine the validity and reliability of the feedback device for transversus abdominis muscle contraction.

\section{Methods:}

The participants in this study were 20 healthy males and females (aged $24.1 \pm 6.8$ years). The feedback sensor was attached to the lumbar support at the front of the trunk. The participants performed an abdominal drawing-in maneuver in order to activate the transversus abdominis muscle, and values from the feedback sensor were collected at the same time. Ultrasound imaging of the transversus abdominis muscle was also collected simultaneously. The feedback sensor collected values at different clinical levels of the pressure biofeedback unit at $64,66,68$, and $70 \mathrm{mmHg}$. The protocol was repeated with a 24-hr interval. Intra-class correlation coefficient, coefficient of variation and standard error of measurements were used to examine reliability. The validity of the values obtained from the relationship between the feedback sensor and transversus abdominis muscle thickness was analyzed using Pearson's correlation coefficients.

Results:

Test-retest reliability of the feedback sensor was excellent $(\mathrm{ICC}=0.946, \mathrm{CV}=2.6 \%, \mathrm{SEMs}=0.54 \%)$. Values of the feedback sensor reported a significantly moderate correlation with the gold standard ultrasound measurement $(\mathrm{r}=-0.514, p<0.001)$.

\section{Conclusion:}

The feedback device demonstrated potential reliability and validity for clinical use by indicating activation of the transversus abdominis muscle.

Keywords: Validity, Reliability, Transversus abdominis, Feedback, Sensor, Core muscles, Ultrasound.

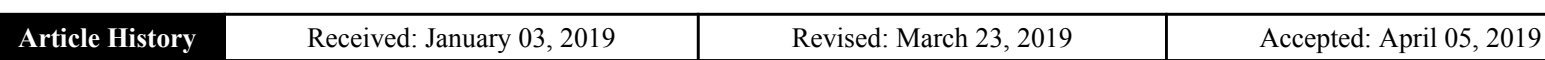

\section{INTRODUCTION}

The Transversus Abdominis (TrA) muscle has been considered an important component of lumbopelvic stability [1,2]. Previous studies [1, 3 - 5] demonstrated that the TrA was activated prior to limb movement, and its automatic contraction occurred during postural changes, which increased spinal stabi-

\footnotetext{
* Address correspondence to these authors at the Department of Physical Therapy, Faculty of Associated Medical Sciences, Chiang Mai University, Chiang Mai, Thailand; Tel: +66089422366; Fax: +66 053946042;

E-mails: aatit.p@cmu.ac.th, patraporn.s@cmu.ac.th
}

lity in asymptomatic individuals. Adults with Low Back Pain (LBP) showed a delayed contraction of the TrA during limb movement and less activation of it while performing postural changes [5]. The presence of LBP suggested an impaired neuromuscular system for controlling and protecting the spine [6]. Therefore, TrA muscle training is suitable for preventing and managing LBP.

Core stability exercise is an effective intervention for reducing pain and recurrence in LBP patients [7, 8]. Abdominal Drawing in Maneuver (ADIM) is a technique that pro- 
motes co-contraction of the TrA and lumbar multifidus muscle [9]. It was proved that activation of the TrA muscle increased the stability of the spine, due to improved motor control, increased abdominal pressure and tightened thoracolumbar fascia [10 - 12]. LBP patients, who were prescribed core stability exercise, demonstrated improvement of pain and disability associated with their condition $[13,14]$.

There are various feedback methods used to indicate $\operatorname{Tr} A$ activation in research and clinical settings such as Electromyography (EMG), ultrasonography, Pressure Biofeedback Unit (PBU) and palpation [15 - 18]. EMG is an invasive method that is limited in its adoption [19]. Lee and colleagues [15] compared the effectiveness of three feedback methods (i.e. ultrasonography, $\mathrm{PBU}$, and palpation) for contracting the TrA muscle and found that using visual feedback was more effective than only palpation. However, there was a limitation in using visual feedback methods while performing functional tasks or during activity of daily living. Therefore, clinicians need an affordable, reliable, and valid alternative tool to deal with TrA activation during functional positions. This study intended to develop a sensor tool for the detection and feedback of core muscle activation while wearing lumbar support, which is portable and easy to use. However, this new device needed to be proven for its reliability and validity.

Therefore, the purpose of this study was to determine the validity and reliability of a Feedback Sensor (FS) device for detecting TrA muscle contraction using real-time ultrasound imaging as a gold standard comparative tool.

\section{MATERIALS AND METHODS}

The protocol was approved by the Institutional Ethics Com-mittee according to the Declaration of Helsinki principles. All participants gave written informed consent before the study began.

\subsection{Participants}

Twenty healthy male and female subjects (aged $24.1 \pm 6.8$ years; 7 males, 13 females, height $164.5 \pm 9.1 \mathrm{~cm}$. and weight $64.2 \pm 8 \mathrm{~kg}$.) participated in this study. They were recruited from the university and areas of the community from December 2017 to January 2018. Those with a history of LBP during the previous three months, lumbar surgery, pregnancy, neuromuscular or joint disease or neurological conditions affecting the trunk were excluded.

\subsection{Development of the Feedback Sensor (FS) Unit}

The FS unit was designed and developed using a pressure sensor. Once built, it was attached to the inner site of the lumbar support. Decreased value of FS pressure was interpreted as increased activation of the TrA.

\subsection{Procedure}

All of the participants were instructed to perform the ADIM using the PBU (Chattanooga Group Inc., USA) as a feedback tool.

The FS was applied at the front of the TrA muscle [1 inch above the Anterior Superior Iliac Spine (ASIS) and 4 inches lateral to the umbilicus], but underneath the lumbar support. One PBU was placed centrally between the umbilicus and the lumbar support and another between the lumbopelvic region (upper border at the L2 level) and lumbar support. The lumbar support was worn firmly, and the PBU at the front was pumped to $70 \mathrm{mmHg}$, and that at the back to $40 \mathrm{mmHg}$. The participants were positioned standing upright, with the trunk stabilized by the wall. They achieved core muscle activation in the functional standing position by performing the ADIM at various levels of pressure (i.e., 70, 68, 66, and $64 \mathrm{mmHg}$, consecutively). Pressure of the PBU at the back was maintained at $40 \mathrm{mmHg}$ for controlling the neutral spinal position. Values from the FS and images of the TrA muscle were collected simultaneously while the ADIM was performed at each level. Decreased sensor values indicated the amount of TrA muscle activation. The participants were allowed to rest for 1 minute between each trial. Each level of pressure and the image were collected three times. The average value was calculated. The participants were also scheduled for two sessions of reliability study with a 24 -hour interval between them.

\subsection{Outcome Measurements}

Real-time ultrasound imaging was used to investigate the amount of TrA muscle contraction during various levels of ADIM performed against the force production from the FS attached to the lumbar support. The ultrasound scanner (Toshiba, Famio 8, SSA-530A) in B-mode, with a 5-MHz curvilinear transducer, was used to assess TrA muscle thickness. The participants were positioned standing in an upright position. Ultrasonic gel was applied between the transducer and skin. The transducer was placed in the transverse plane at a point $2.5 \mathrm{~cm}$ anteromedial to the midpoint between the lower rib and iliac crest on the mid-axillary line [19]. The image was captured at the end of exhalation. After that, muscle thickness was measured during TrA activation at each pressure level of the PBU. Thickness of the TrA was measured randomly on both sides. Images and measurements of the TrA were obtained using NIH (Bethesda, MD) Image J software (V 1.8). The mean thickness of three measurements on each side was calculated.

\subsection{Statistical Analysis}

The test-retest reliability of force production was determined using Intra-Class Correlation Coefficients (ICCs). Coefficient of Variation (CV) and Standard Error of Measurements (SEMs) were also included for determining variability of measurements. The measurements were determined as accep-tably reliable if the ICC value was greater than $0.85, \mathrm{CV}$ was less than $15 \%$, and SEMs were less than $5 \%$ $[20,21]$. The validity of the values obtained from the relationship between the FS and TrA thickness was analyzed using Pearson's co-rrelation coefficients. Statistical analysis was performed using the Statistical Package for the Social Sciences (SPSS).

\section{RESULTS}

Table 1 shows the ICCs, CV and SEMs for all measurements. The FS and measurement of TrA thickness were considered as being acceptably reliable (i.e. ICC $>0.9, \mathrm{CV}<10 \%$, SEMs $<5 \%$ ). 
Table 1. The test - retest reliability results of the feedback sensor device and the real time ultrasound imaging of TrA thickness.

\begin{tabular}{|c|c|c|c|}
\hline Measurements & ICC & \%CV & SEMs \\
\hline Feedback Sensor & 0.946 & 2.6 & $2.47(0.54 \%)$ \\
\hline TrA thickness & 0.931 & 8.05 & $0.104(2.09 \%)$ \\
\hline
\end{tabular}

ICC: Intraclass Correlation Coefficients; CV: Coefficient of Variation; SEMs: Standard Error of Measurements

Table 2. Correlation between feedback sensor device and the thickness of transversus abdominis muscle.

\begin{tabular}{|c|c|c|}
\hline Measurements & Pearson's Correlation Coefficient (r) \\
\hline PBU $v s$ Feedback Sensor device & 0.657 & $<0.001$ \\
\hline PBU $v s$ TrA thickness & -0.793 & $<0.001$ \\
\hline Feedback Sensor device $v s$ TrA thickness & -0.514 & $<0.001$ \\
\hline
\end{tabular}

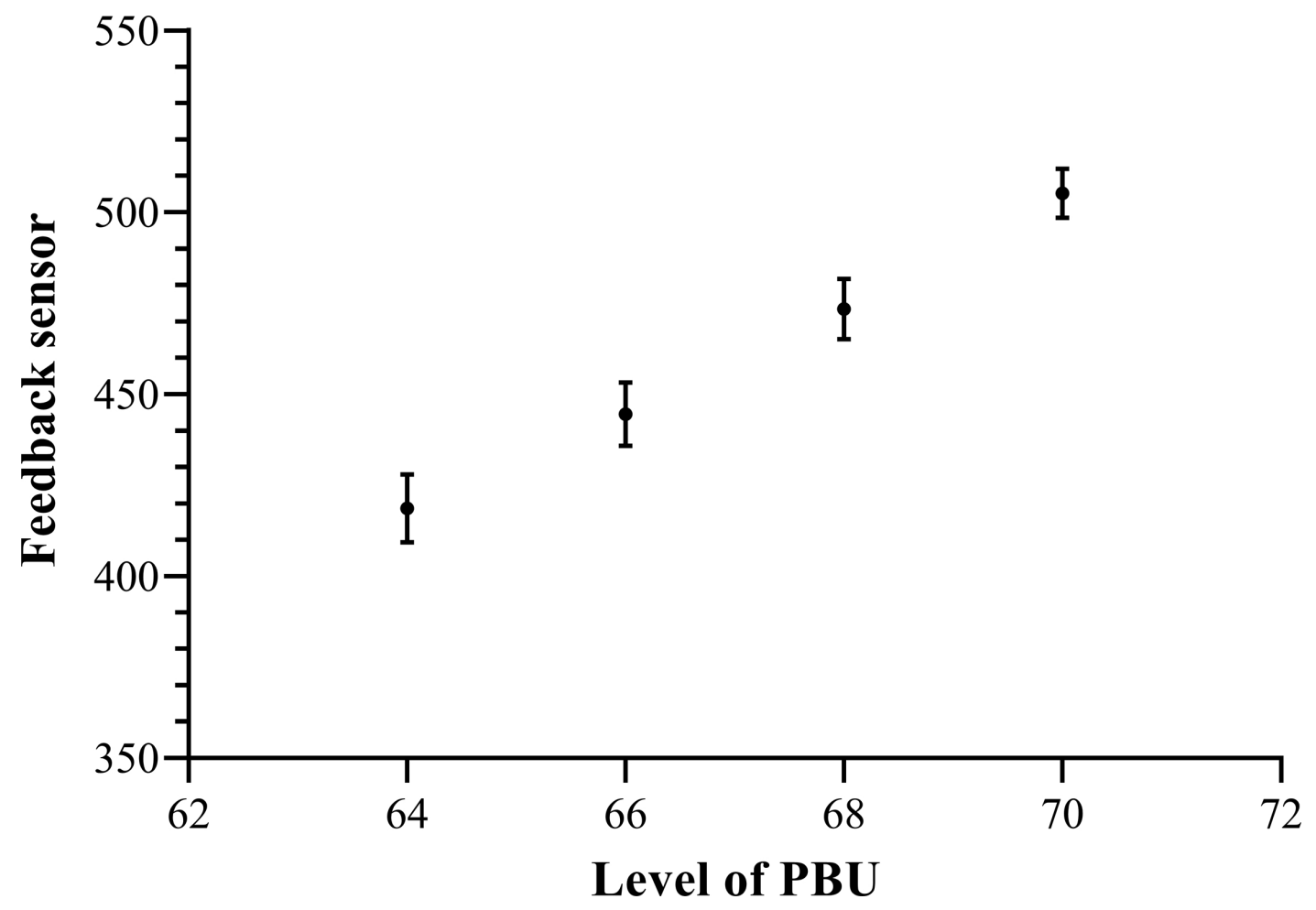

Fig. (1). Relationship between levels of the PBU and FS device.

Results of FS validity for activating the TrA muscle are presented in Table 2. There was a significant correlation (moderately positive) between the PBU and FS $(r=0.657, p<$ 0.001). Value of the FS at each level of the PBU is shown in Fig. (1). There was a strong negative correlation between the PBU and TrA thickness $(r=-0.793, p<0.001)$. Thickness of the TrA at each level of the PBU is shown in Fig. (2). There was a significant correlation (moderately negative) between the FS and TrA thickness $(r=-0.514, p<0.001)$. The correlation between the FS and TrA thickness is shown in Fig. (3).

\section{DISCUSSION}

The purpose of this study was to evaluate the reliability and validity of the feedback device compared with the ultrasound imaging for activating the TrA muscle, and report test-retest reliability of the device. This study found excellent reliability of the FS and measurement of the TrA thickness using ultrasound imaging in a standing position (ICC $=0.946$ and 0.931 , respectively). This suggested that the FS and measurement method of TrA thickness in this study were reliable methods. Previous studies $[9,22]$ also found very high reliability in ultrasound measurement of the TrA muscle (ICC $>0.9$ ) in supine position. The high Intra-Class Correlation of TrA thickness consistency in an upright position correlated with the result in this study of that in a standing position (ICC $>0.9)$ [23].

A significant (moderate) relationship was found between the FS and thickness of the TrA. Utilizing the FS in this study could be considered in order to give information of TrA activa- 


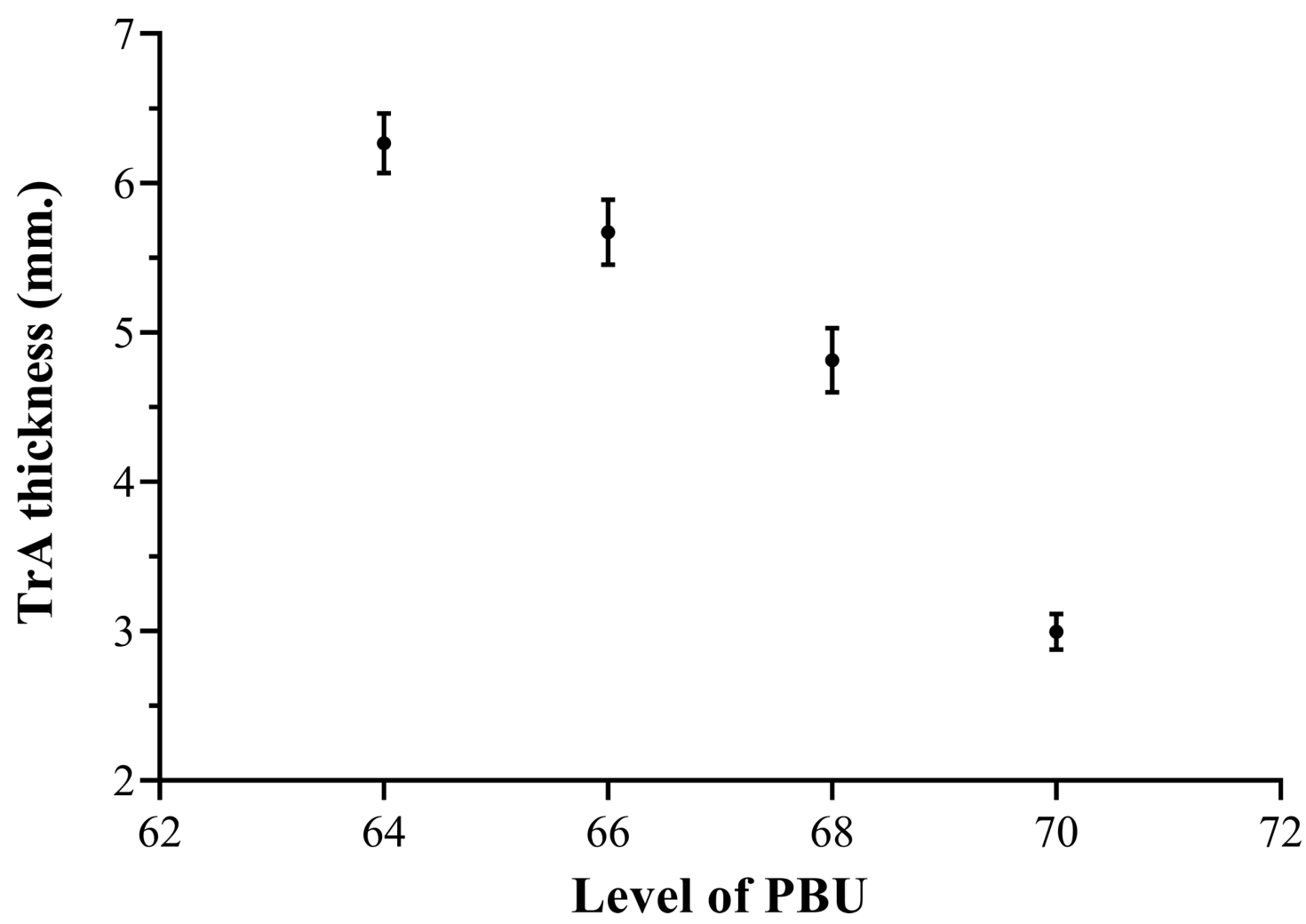

Fig. (2). Relationship between levels of the PBU and thickness of the TrA muscle.

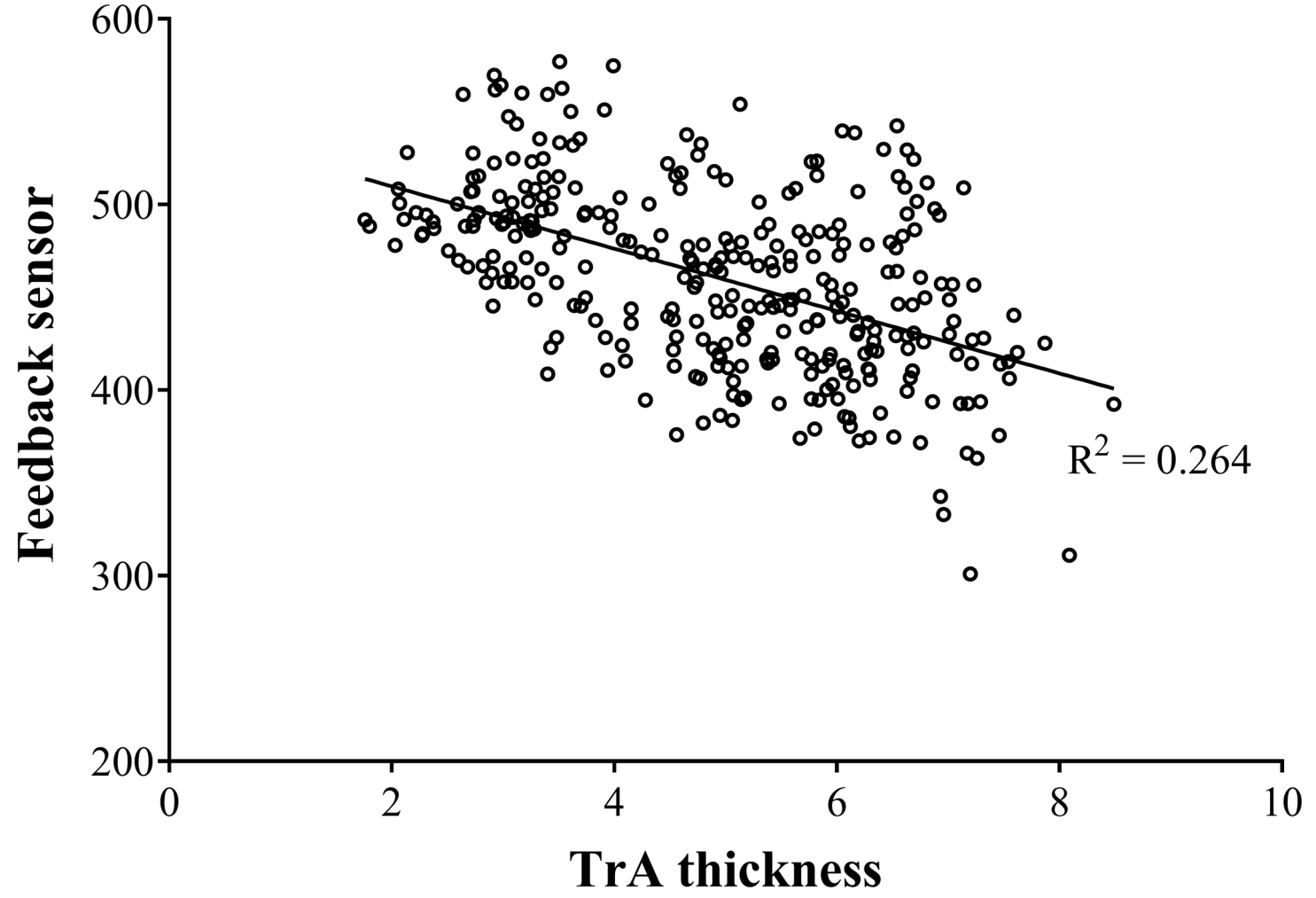

Fig. (3). Relationship between the FS device and thickness of the TrA muscle. 
tion during the performance of ADIM in a functional upright position. The negative relationship between the FS and TrA thickness was as expected. The FS was put between the lumbar support and abdominal muscles. When performing the ADIM, the pressure values from the FS and PBU, which were located between the lumbar support and abdominal muscles, were decreased. At the same time, the TrA was activated by performing the ADIM, and presented in the increase of muscle thickness. This was supported by the study of McMeekan et al. [19], which showed an excellent correlation between the activity and thickness of the TrA. In addition, Lee and colleagues [15] reported that ADIM training with the feedback method (i.e. ultrasound imaging and $\mathrm{PBU}$ ) for 15 minutes resulted in significantly thicker TrA muscle than using manual contact. They suggested that use of the feedback method may be more effective only in improving TrA muscle function than using manual contact. Therefore, the feedback device in this study may be useful as an indicator of TrA activation for patients with LBP, especially for self-training at home or the workplace because it is easy to use and affordable.

\section{CONCLUSION}

This study demonstrated that the feedback device, which was developed in this study, is considered as a reliable and valid tool for providing information on TrA activation during performance of the ADIM. It could be a clinically useful device for simultaneous feedback in activating the TrA muscle and encouraging patients with LBP to perform precise core stability activation.

A limitation of this study was validity and reliability of the feedback device being evaluated in healthy subjects in a standing position. Further study may require various patient populations and different positions during activity of daily living.

\section{LIST OF ABBREVIATIONS}

$\begin{array}{lll}\text { LBP } & =\text { Low Back Pain } \\ \text { FS } & =\text { Feedback Sensor } \\ \text { TrA } & =\text { Transversus Abdominis } \\ \text { ADIM } & =\text { Abdominal Drawing-in Maneuver } \\ \text { EMG } & =\text { Electromyography } \\ \text { PBU } & =\text { Pressure Biofeedback Unit } \\ \text { ASIS } & =\text { Anterior Superior Iliac Spine }\end{array}$

\section{ETHICS APPROVAL AND CONSENT TO PARTICIPATE}

Ethical approval was obtained by the Institutional Ethics Committee with approval number AMSEC-60EX-028.

\section{HUMAN AND ANIMAL RIGHTS}

No animals were used in this research. All human research procedures followed were in accordance with the ethical standards of the committee responsible for human experimentation (institutional and national), and with the Helsinki Declaration of 1975, as revised in 2013.

\section{CONSENT FOR PUBLICATION}

All participants gave written informed consent.

\section{AVAILABILITY OF DATA AND MATERIALS}

Not applicable.

\section{FUNDING}

This study was supported by Thailand Research Fund, grant number TRF-RRi 59I0017.

\section{CONFLICT OF INTEREST}

The authors declare no conflict of interest, financial or otherwise.

\section{ACKNOWLEDGEMENTS}

The authors are pleased to acknowledge the support of Thailand Research Fund.

\section{REFERENCES}

[1] P.W. Hodges, and C.A. Richardson, "Delayed postural contraction of transversus abdominis in low back pain associated with movement of the lower limb", J. Spinal Disord., vol. 11, no. 1, pp. 46-56, 1998. [http://dx.doi.org/10.1097/00002517-199802000-00008] [PMID: 949 3770]

[2] V. Akuthota, A. Ferreiro, T. Moore, and M. Fredericson, "Core stability exercise principles", Curr. Sports Med. Rep., vol. 7, no. 1, pp. 39-44, 2008.

[http://dx.doi.org/10.1097/01.CSMR.0000308663.13278.69] [PMID: 18296944]

[3] P.W. Hodges, and C.A. Richardson, "Contraction of the abdominal muscles associated with movement of the lower limb", Phys. Ther., vol. 77, no. 2, pp. 132-142, 1997.

[http://dx.doi.org/10.1093/ptj/77.2.132] [PMID: 9037214]

[4] O. Vasseljen, M. Unsgaard-Tøndel, C. Westad, and P.J. Mork, "Effect of core stability exercises on feed-forward activation of deep abdominal muscles in chronic low back pain: A randomized controlled trial", Spine, vol. 37, no. 13, pp. 1101-1108, 2012.

[http://dx.doi.org/10.1097/BRS.0b013e318241377c] [PMID: 221462 80]

[5] T. Miura, M. Yamanaka, K. Ukishiro, H. Tohyama, H. Saito, M. Samukawa, T. Kobayashi, T. Ino, and N. Takeda, "Individuals with chronic low back pain do not modulate the level of transversus abdominis muscle contraction across different postures", Man. Ther., vol. 19 , no. 6 , pp. 534-540, 2014.

[http://dx.doi.org/10.1016/j.math.2014.05.010] [PMID: 25009124]

[6] E. Lederman, "The myth of core stability", J. Bodyw. Mov. Ther., vol. 14, no. 1, pp. 84-98, 2010.

[http://dx.doi.org/10.1016/j.jbmt.2009.08.001] [PMID: 20006294]

[7] G.E. Hicks, J.M. Fritz, A. Delitto, and S.M. McGill, "Preliminary development of a clinical prediction rule for determining which patients with low back pain will respond to a stabilization exercise program", Arch. Phys. Med. Rehabil., vol. 86, no. 9, pp. 1753-1762, 2005.

[http://dx.doi.org/10.1016/j.apmr.2005.03.033] [PMID: 16181938]

[8] C. Demoulin, V. Distrée, M. Tomasella, J-M. Crielaard, and M. Vanderthommen, "Lumbar functional instability: A critical appraisal of the literature", Ann. Readapt. Med. Phys., vol. 50, no. 8, pp. 677-684, 669-676, 2007.

[http://dx.doi.org/10.1016/j.annrmp.2007.05.007] [PMID: 17597247]

[9] D.S. Teyhen, C.E. Miltenberger, H.M. Deiters, Y.M. Del Toro, J.N. Pulliam, J.D. Childs, R.E. Boyles, and T.W. Flynn, "The use of ultrasound imaging of the abdominal drawing-in maneuver in subjects with low back pain", J. Orthop. Sports Phys. Ther., vol. 35, no. 6, pp. 346-355, 2005.

[http://dx.doi.org/10.2519/jospt.2005.35.6.346] [PMID: 16001906]

[10] P.W. Hodges, "Is there a role for transversus abdominis in lumbopelvic stability?", Man. Ther., vol. 4, no. 2, pp. 74-86, 1999. [http://dx.doi.org/10.1054/math.1999.0169] [PMID: 10509061]

[11] J. Cholewicki, K. Juluru, and S.M. McGill, "Intra-abdominal pressure 
mechanism for stabilizing the lumbar spine", J. Biomech., vol. 32, no. 1, pp. 13-17, 1999.

[http://dx.doi.org/10.1016/S0021-9290(98)00129-8] [PMID: 100509 47]

[12] A.G. Cresswell, L. Oddsson, and A. Thorstensson, "The influence of sudden perturbations on trunk muscle activity and intra-abdominal pressure while standing", Exp. Brain Res., vol. 98, no. 2, pp. 336-341, 1994.

[http://dx.doi.org/10.1007/BF00228421] [PMID: 8050518]

[13] J.A. Hides, G.A. Jull, and C.A. Richardson, "Long-term effects of specific stabilizing exercises for first-episode low back pain", Spine, vol. 26, no. 11, pp. E243-E248, 2001

[http://dx.doi.org/10.1097/00007632-200106010-00004] [PMID: 1138 9408]

[14] X-Q. Wang, J-J. Zheng, Z-W. Yu, X. Bi, S-J. Lou, J. Liu, B. Cai, Y.H. Hua, M. Wu, M.L. Wei, H.M. Shen, Y. Chen, Y.J. Pan, G.H. Xu, and P.J. Chen, "A meta-analysis of core stability exercise versus general exercise for chronic low back pain", PLoS One, vol. 7, no. 12, p. e52082, 2012.

[http://dx.doi.org/10.1371/journal.pone.0052082] [PMID: 23284879]

[15] S. Lee, S. Han, and D. Lee, "Comparison of abdominal muscle thickness according to feedback method used during abdominal hollowing exercise", J. Phys. Ther. Sci., vol. 28, no. 9, pp. 2519-2521, 2016. [http://dx.doi.org/10.1589/jpts.28.2519] [PMID: 27799683]

[16] K-H. Yang, and D-J. Park, "Reliability of ultrasound in combination with surface electromyogram for evaluating the activity of abdominal muscles in individuals with and without low back pain", J. Exerc. Rehabil., vol. 10, no. 4, pp. 230-235, 2014.

[http://dx.doi.org/10.12965/jer.140138] [PMID: 25210698]

[17] S. Uddin, and F. Ahmed, Effect of lumbar stabilization exercises versus pressure feedback training in low back ache patients. Eur. Sci. J., vol. 9. ESJ, 2013

[18] S.M. Henry, and K.C. Westervelt, "The use of real-time ultrasound feedback in teaching abdominal hollowing exercises to healthy subjects", J. Orthop. Sports Phys. Ther., vol. 35, no. 6, pp. 338-345, 2005.

[http://dx.doi.org/10.2519/jospt.2005.35.6.338] [PMID: 16001905]

[19] J.M. McMeeken, I.D. Beith, D.J. Newham, P. Milligan, and D.J. Critchley, "The relationship between EMG and change in thickness of transversus abdominis", Clin. Biomech. (Bristol, Avon), vol. 19, no. 4, pp. 337-342, 2004.

[http://dx.doi.org/10.1016/j.clinbiomech.2004.01.007] [PMID: 15109 752]

[20] G. Atkinson, and A.M. Nevill, "Statistical methods for assessing measurement error (reliability) in variables relevant to sports medicine", Sports Med., vol. 26, no. 4, pp. 217-238, 1998.

[http://dx.doi.org/10.2165/00007256-199826040-00002] [PMID: 982 0922]

[21] L.G. Portney, Foundations of clinical research: Applications to practice 3th (third)., Edition, 2009.

[22] J-R. Jeong, J.H. Han, J-E. Cho, and W-h. Lee, "Reliability and validity of a personal computer based muscle viewer for measuring upper trapezius and transverses abdominis muscle thickness", Phys. Ther. Rehabil. Sci., vol. 5, pp. 155-161, 2016.

[http://dx.doi.org/10.14474/ptrs.2016.5.3.155]

[23] C.W. Hoppes, A.D. Sperier, C.F. Hopkins, B.D. Griffiths, M.F. Principe, B.L. Schnall, J.C. Bell, and S.L. Koppenhaver, "Ultrasound imaging measurement of the transversus abdominis in supine, standing, and under loading: A reliability study of novice examiners", Int. J. Sports Phys. Ther., vol. 10, no. 6, pp. 910-917, 2015. [PMID: 26618070]

(C) 2019 Dissanguan et al.

This is an open access article distributed under the terms of the Creative Commons Attribution 4.0 International Public License (CC-BY 4.0), a copy of which is available at: (https://creativecommons.org/licenses/by/4.0/legalcode). This license permits unrestricted use, distribution, and reproduction in any medium, provided the original author and source are credited. 